\title{
Management of Premature Infants Using the Kangaroo Method versus the Classic Method: Morbidity and Prognosis Associated with in Sendwe General Hospital in Lubumbashi (DR Congo)
}

\author{
Judith Sangwa Sinanduku1, Gray Kanteng1*, Franck Moma², \\ Stanis Wembonyama Okitotsho ${ }^{1}$, Oscar Luboya ${ }^{1}$ \\ ${ }^{1}$ Département de Pédiatrie, Université de Lubumbashi, Lubumbashi, Congo \\ ${ }^{2}$ Ministère de la Santé Publique, Inspection provincial de la Santé du Haut-Katanga, Lubumbashi, Congo \\ Email: *gkanteng@yahoo.fr
}

How to cite this paper: Sinanduku, J.S., Kanteng, G., Moma, F., Okitotsho, S.W. and Luboya, O. (2019) Management of Premature Infants Using the Kangaroo Method versus the Classic Method: Morbidity and Prognosis Associated with in Sendwe General Hospital in Lubumbashi (DR Congo). Open Access Library Journal, 6: e5150

https://doi.org/10.4236/oalib.1105150

Received: December 28, 2018

Accepted: January 28, 2019

Published: January 31, 2019

Copyright $\odot 2019$ by author(s) and Open Access Library Inc.

This work is licensed under the Creative Commons Attribution International License (CC BY 4.0).

http://creativecommons.org/licenses/by/4.0/

\section{(c) (i) Open Access}

\begin{abstract}
Introduction: This study aims to compare the benefit of two low low-birth weight newborn management methods, namely the "classic" method using incubators, and the Kangaroo method in a hospital setting; and determine the survival prognosis associated with each of these methods. Methods: We conducted a longitudinal cohort study from 2013 to 2015 at the Jason Sendwe General Hospital in Lubumbashi (DR Congo). A total of 200 cases of low birth weight infants were included, followed by one or the other of the methods evaluated. Results: The average weight at the exit was better under Kangaroo (2191.8 $\pm 212.1 \mathrm{~g}$ versus $2068.6 \pm 476.5 \mathrm{~g})$ as well as the average gain in weight per day (52.2 $\pm 9.5 \mathrm{~g}$ versus $31.0 \pm 15.1 \mathrm{~g})$. Fewer episodes of hypothermia were noted under Kangaroo, and the duration of stay was shorter $(10.2 \pm 2.9$ days versus $13.0 \pm 5.4$ days). Logistic regression showed that the Kangaroo method is better for the management of premature infants $(\mathrm{p}=0.004, \mathrm{ORa}=4.39(1.60-12.04))$. Survival is significantly higher under the Kangaroo method $(\mathrm{p}<0.001)$. The median survival under Kangaroo versus classical method was 16 (EIQ: 15 - 16) and 10 (EIQ: 9 - 10) months, respectively. The incidence of Kangaroo deaths was 0.3 cases per $100 \mathrm{P}-\mathrm{J}$ versus 1.9 cases per $100 \mathrm{P}-\mathrm{D}(\mathrm{P}<0.001)$ using the conventional method. Conclusion: The Kangaroo method is applicable and offers guarantees of a beneficial management in the newborn with low birth weight.
\end{abstract}




\section{Subject Areas}

Gynecology \& Obstetrics

\section{Keywords}

Premature, Low Birth Weight, Kangaroo Method, Incubator

\section{Introduction}

La prise en charge du prématuré pose un défi majeur, notamment dans les pays à faible revenus. En effet, 2.9 millions d'enfants meurent chaque année dans le monde en période néonatale et, dans un tiers des cas, ces morts sont en relation avec la prématurité et/ou le petit poids de naissance à cause soit des maladies infectieuses, soit à cause de l'immaturité des organes [1]. D'où l'intérêt d'une gestion efficiente de la thermorégulation chez le nouveau-né.

L'utilisation des incubateurs a amélioré la gestion de la thermorégulation du nouveau-né, mais son accessibilité est moindre et son utilisation délicate dans le contexte des pays à faibles ressources. Ainsi, l'intérêt d'une méthode alternative, disponible et à faible coût, à savoir la méthode de soins mère Kangourou (SMK) ou peau à peau, a vite émergé et s'impose aujourd'hui comme un moyen efficace de prise en charge de l'hypothermie du prématuré. Elle permet une utilisation rationnelle des ressources humaines et technologiques disponibles, en particulier quand elles sont insuffisantes dans les pays en voie de développement [2]. Les soins mère-kangourou ( $\mathrm{SMK}$ ) sont une excellente alternative de soins pour le bébé prématuré ou de faible poids de naissance, mais seulement si certaines règles sont respectées.

Plusieurs travaux attestent de l'efficacité de cette méthode dans la prise en charge du faible poids de naissance. Une étude menée au Canada montre que la méthode n'a relevé aucun effet néfaste sur la stabilité physiologique des nourrissons dès 26 semaines d'âge gestationnel, même lorsqu'ils étaient sous ventilation assistée; et que les mères présentent des comportements d'attachement plus marqués et une meilleure perception de leur rôle de mère [3]. A Niamey (Niger), une étude a constaté une baisse de la mortalité, une diminution de la durée de séjour ainsi qu'une meilleure prise de poids chez les nouveau-nés de faible poids de naissance lorsqu'ils sont pris en charge par la méthode SMK [4].

Aujourd'hui, même dans un contexte où les moyens techniques de prise en charge classique du nouveau-né à l'aide d'incubateurs sont disponibles, il semble de plus en plus avéré que la méthode kangourou présente des avantages. C'est ainsi que cette étude s'est fixée comme objectif de comparer le bénéfice des deux méthodes de prise en charge du prématuré, à savoir la méthode "classique" utilisant des incubateurs, et la méthode Kangourou dans un hôpital général de référence en $\mathrm{RD}$ Congo; et de déterminer le pronostic de survie associé à chacune de ces méthodes. 


\section{Methodologie}

\subsection{Cadre D'étude}

La présente étude a été menée en $\mathrm{RD}$ Congo, dans la ville de Lubumbashi, à l'hôpital Jason Sendwe. Cet hôpital est une structure du $3^{\text {ème }}$ échelon selon le système de soins du pays, soit le niveau le plus élevé. Il a une vocation d'hôpital général provincial de référence, et possède également le statut de d'hôpital de formation universitaire.

Le travail s'est déroulé au sein du service de néonatologie, qui a une capacité de 6 couveuses. L'unité SMK a été créée en 2013, au sein du service de néonatologie. Ce service est attenant à la maternité et au bloc opératoire; il reçoit les nouveau-nés transférés des maternités de la ville et qui nécessitent un traitement en soins intensifs, mais aussi ceux qui sont nés au sein même de l'hôpital. Le plateau technique permet d'assurer les soins essentiels du nouveau-né, mais également certains soins complexes comme une oxygénothérapie, une photothérapie, etc. Le service bénéficie de l'expertise de médecins spécialistes en pédiatrie, de médecins généralistes et d'infirmiers habilités dans la prise en charge du nouveau-né.

\subsection{Type et Durée D'étude}

Nous avons mené une étude longitudinale de cohorte sur une période de 3 ans, soit de 2013 à 2015.

\subsection{Echantillonnage}

- Type d'échantillon: Notre échantillonnage était exhaustif, incluant tous les cas répondant aux critères d'inclusion décrits plus bas.

- Taille de l'échantillon: Au total, 200 cas ont été inclus dans l'étude.

- Critères d'inclusion: Ont été inclus tous les nouveau-nés de faible poids de naissance hospitalisés dans le service de néonatologie durant la période d'étude, soit ayant un poids de moins de 2000 gr, né à terme ou prématurément.

- Critères de non inclusion: Ont été exclus tous les nouveau-nés avec un poids de plus de 2000 grammes et ceux décédés endéans les premières 24 heures.

\subsection{Technique de Collecte de Données}

Deux cohortes de nouveau-nés ont été simultanément suivies, depuis l'admission jusqu'à la sortie (et/ou le décès). La première concernait les nouveau-nés de faible poids de naissance dont la thermorégulation était exclusivement assurée par la méthode classique, c'est-à-dire au moyen d'un incubateur. La deuxième était composée des nouveau-nés suivis par la méthode de soins mère kangourou. Les critères d'éligibilité à la méthode de soins mère kangourou étaient: l'acceptation de la mère (accompagnatrice) et l'autonomie clinique (sur le plan cardiorespiratoire notamment). 


\subsection{Analyses Statistiques}

Les analyses de données ont été réalisées à l'aide des logiciels SPSS version 21.

Les statistiques descriptives ont été présentées sous forme de moyenne majorée de l'écart-type pour l'âge de la mère, la parité, la gestité, l'EIG, poids du nouveau-né, taille du nouveau-né, périmètre crânien, périmètre brachial et gain pondéral journalier et en fréquence absolue et pourcentages pour les données catégorielles comme les caractéristiques sociodémographiques et cliniques des mère; les caractéristiques cliniques des prématurés, l'issue vitale.

Les comparaisons entre le groupe de Kangourou et classique ont été faites selon le cas, à l'aide, respectivement de test t-Student pour les variables quantitatives; le test de chi carré ou le test exact de Fisher pour les variables catégorielles.

Le test de régression logistique a permis de rechercher les facteurs associés à la bonne croissance de prématurés; la bonne croissance a été défini pour tout prématuré ayant atteint $2500 \mathrm{~g}$ à la sortie, l'odd ratio calculé a permis d'estimé cette association.

La méthode de Kaplan Meier a permis de décrire la survie entre la date de l'initiation de la prise en charge des patients et le décès (données complètes) et la fin de l'étude (données censurées). Le test de Log-rank a été utilisé pour comparer les courbes de survie. La régression de Cox a recherché les prédicteurs indépendants de la mortalité chez ces prématurés. Une valeur de $\mathrm{p}<$ à 0.05 a été considérée comme le seuil de significativité statistique.

\subsection{Considérations Éthiques}

Nous avons recouru à un consentement éclairé verbal de chaque mère avant de requérir son avis pour l'inclusion dans l'étude. Un accord verbal était obtenu de chaque participant avant son inclusion.

\section{Resultats}

Deux cents prématurés ont fait l'objet de l'étude élevés selon deux méthodes 78 (39\%) sous la méthode kangourou et 122 (61\%) sous la méthode classique.

\subsection{Caractéristiques des Mères}

Il ressort du Table 1 que les nouveau-nés suivis par la méthode Kangourou sont significativement associés aux mères ayant moins de complications et dont la voie d'accouchement était la voie basse.

\subsection{Caractéristiques des Nouveau-Nés}

Le Table 2 montre une association significative entre la méthode SMK et une meilleure prise de poids à la sortie, un meilleur gain de poids et du périmètre thoracique, un meilleur gain de poids par jour, une meilleure croissance, moins d'épisodes d'hypothermie et d'hyperthermie, et une durée de séjour plus courte.

\subsection{Facteurs Associés à la Bonne Croissance de Prématurés}

Selon le Table 3, l'analyse multivariée a montré les facteurs significatifs ci-après 
associés à la bonne croissance des prématurés: un poids supérieur ou égal à 1600 g, une alimentation au lait maternel et un élevage par la méthode SMK.

\subsection{Survie des Prématurés}

\section{1) Issue vitale}

Après le premier jour d'admission, le nombre de décès était de 32 (16\%). Après

Table 1. Répartition des mères selon la méthode de prise en charge.

\begin{tabular}{|c|c|c|c|c|}
\hline Variables & $\begin{array}{c}\text { Tous } \\
\mathrm{n}=200\end{array}$ & $\begin{array}{c}\text { Kangourou } \\
\mathrm{n}=78\end{array}$ & $\begin{array}{c}\text { Classique } \\
\mathrm{n}=122\end{array}$ & $\mathrm{p}$ \\
\hline Age & $26.7 \pm 6.1$ & $28.7 \pm 6.0$ & $25.4 \pm 5.8$ & 0.022 \\
\hline$<20$ ans & $33(16.5)$ & $8(10.3)$ & $25(20.5)$ & \\
\hline $20-34$ ans & $140(70.0)$ & $54(69.2)$ & $86(70.5)$ & \\
\hline$\geq 35$ ans & $27(13.5)$ & $16(20.5)$ & $11(9.0)$ & \\
\hline Etat civil & & & & $<0.001$ \\
\hline Célibataire & $32(16.0)$ & $18(23.1)$ & $14(11.5)$ & \\
\hline Mariée & $145(72.5)$ & $59(75.6)$ & $86(70.5)$ & \\
\hline Union libre & $23(11.5)$ & $1(1.3)$ & $22(18.0)$ & \\
\hline Parité & $4.0 \pm 1.1$ & $4.4 \pm 1.2$ & $3.8 \pm 1.9$ & 0.360 \\
\hline $\mathrm{P} 1$ & $31(15.5)$ & $10(12.8)$ & $21(17.2)$ & \\
\hline $\mathrm{P} 2-5$ & $125(62.5)$ & $47(60.3)$ & $78(63.9)$ & \\
\hline$>\mathrm{P} 5$ & $44(22.0)$ & $21(26.9)$ & $23(18.9)$ & \\
\hline Gestité & $3.8 \pm 1.9$ & $4.1 \pm 2.0$ & $3.7 \pm 1.9$ & 0.143 \\
\hline G1 & $34(17.0)$ & $10(12.8)$ & $24(19.7)$ & \\
\hline $\mathrm{G} 2+$ & $166(83.0)$ & $68(87.2)$ & $98(80.3)$ & \\
\hline Avortement & & & & 0.656 \\
\hline Non & $172(86.0)$ & $67(85.9)$ & $105(86.1)$ & \\
\hline$\geq 1$ & $28(14.0)$ & $11(14.1)$ & $17(13.9)$ & \\
\hline EIG & $18.7 \pm 11.0$ & $19.8 \pm 12.4$ & $17.9 \pm 10.0$ & 0.223 \\
\hline$<24$ moins & $106(63.5)$ & $46(67.6)$ & $60(60.6)$ & \\
\hline$\geq 24$ moins & $61(36.5)$ & $22(32.4)$ & $39(39.4)$ & \\
\hline Césarienne & $30(15.0)$ & $10(12.8)$ & $20(16.4)$ & 0.316 \\
\hline Naissance $<2500$ & $190(95.0)$ & 74 (94.9) & $116(95.1)$ & 0.596 \\
\hline Naissance Prématuré & $194(97.0)$ & $74(94.9)$ & $120(98.4)$ & 0.162 \\
\hline Naissance Mort-né & $26(13.0)$ & $9(11.5)$ & $17(13.9)$ & 0.396 \\
\hline Décès au J7 & $37(18.5)$ & $15(19.2)$ & $22(18.0)$ & 0.486 \\
\hline Complications & $150(75.0)$ & $45(57.7)$ & $105(86.1)$ & $<0.001$ \\
\hline Mode d'accouchement & & & & 0.039 \\
\hline Voie basse & $176(88.0)$ & $73(93.6)$ & $103(84.4)$ & \\
\hline Césarienne & $24(12.0)$ & $5(6.4)$ & $19(15.6)$ & \\
\hline
\end{tabular}


Table 2. Répartition des nouveau-nés selon la méthode de prise en charge.

\begin{tabular}{|c|c|c|c|c|}
\hline Variables & $\begin{array}{c}\text { Tous } \\
\mathrm{n}=\mathbf{2 0 0}\end{array}$ & $\begin{array}{c}\text { Kangourou } \\
\mathrm{n}=78\end{array}$ & $\begin{array}{c}\text { Classique } \\
\mathrm{n}=122\end{array}$ & $\mathrm{p}$ \\
\hline Sexe & & & & 0.507 \\
\hline Masculin & $96(48.0)$ & $37(47.4)$ & $59(48.4)$ & \\
\hline Féminin & $104(52.0)$ & $41(52.6)$ & $63(51.6)$ & \\
\hline Poids & $1637.2 \pm 282.4$ & $1669.7 \pm \pm 253.1$ & $1616.4 \pm 298.8$ & 0.194 \\
\hline Poids à la sortie & $2116.6 \pm 398.9$ & $2191.8 \pm 212.1$ & $2068.6 \pm 476.5$ & 0.003 \\
\hline Taille & $40.5 \pm 3.3$ & $41.5 \pm 3.3$ & $39.8 \pm 3.2$ & $<0.001$ \\
\hline PC & $30.6 \pm 2.9$ & $30.1 \pm 2.6$ & $30.8 \pm 3.1$ & 0.093 \\
\hline PT & $28.9 \pm 2.7$ & $28.3 \pm 2.5$ & $29.3 \pm 2.8$ & 0.013 \\
\hline $\mathrm{PB}$ & $8.2 \pm 0.8$ & $8.3 \pm 0$ & $8.1 \pm 0.8$ & 0.169 \\
\hline Gain poids moyen par Jour & $39.3 \pm 16.8$ & $52.2 \pm 9.5$ & $31.0 \pm 15.1$ & $<0.001$ \\
\hline Type alimentation & & & & $<0.001$ \\
\hline LM & $98(49.0)$ & $49(62.8)$ & $49(40.2)$ & \\
\hline LA & $2(1.0)$ & $2(2.6)$ & $0(0.0)$ & \\
\hline Mixte & $100(50.0)$ & $27(34.6)$ & $73(59.8)$ & \\
\hline Croissance de prématuré & & & & 0.012 \\
\hline Bonne & $111(55.5)$ & $61(78.2)$ & $50(41.0)$ & \\
\hline Mauvais & $89(44.5)$ & $17(21.8)$ & $72(59.0)$ & \\
\hline Episode d'hypothermie & $154(77.0)$ & $33(42.3)$ & $121(99.2)$ & $<0.001$ \\
\hline Episode d'hyperthermie & $59(29.5)$ & $4(5.1)$ & $55(45.1)$ & $<0.001$ \\
\hline Produit reçu & & & & $<0.001$ \\
\hline ATB & $182(91.0)$ & $62(79.5)$ & $120(98.4)$ & \\
\hline Rien & $18(9.0)$ & $16(20.5)$ & $2(1.6)$ & \\
\hline Durée séjour & $11.9 \pm 4.8$ & $10.2 \pm 2.9$ & $13.0 \pm 5.4$ & $<0.001$ \\
\hline
\end{tabular}

une durée médiane de suivi de 4 jours (EIQ = 3-5) avec 147 personne-jour (P-J); 32 prématurés étaient décédés soit un taux de mortalité de 21,8 décès pour 100 P-J à la date de point.

\section{2) Survie globale}

Selon la Figure 1, la probabilité de survie des prématurés à l'initiation de prise en charge était, respectivement, de $87 \%, 85 \%$ et $84 \%$ à cinq jours, à dix jours et à vingt jours. La période critique était les cinq premiers jours de prise en charge avec $13 \%$ de décès.

\section{3) Survie par sous-groupe}

Il ressort de la Figure 2 sur la comparaison des courbes de survie des prématurés en fonction de la méthode d'élevage, que la survie était significativement ( $\mathrm{p}$ $<0.001$ ) plus élevée chez les prématurés sous méthode Kangourou que chez ceux sous la méthode classique. La survie médiane des prématurés sous Kangourou et 
Table 3. Facteurs associés à la bonne croissance de prématurés.

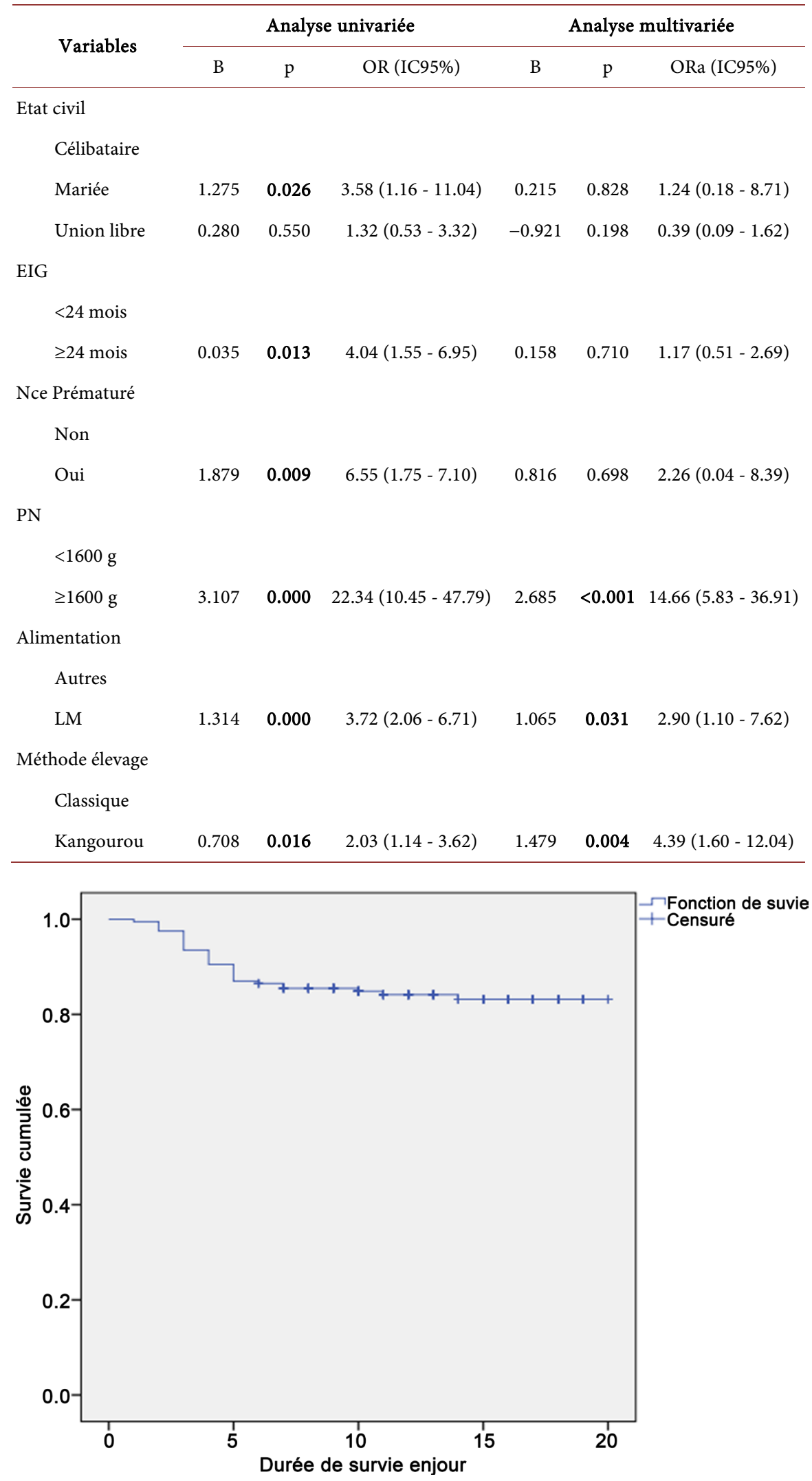

Figure 1. Courbe de survie (Kaplan-Meier) du groupe entier des prématurés. 


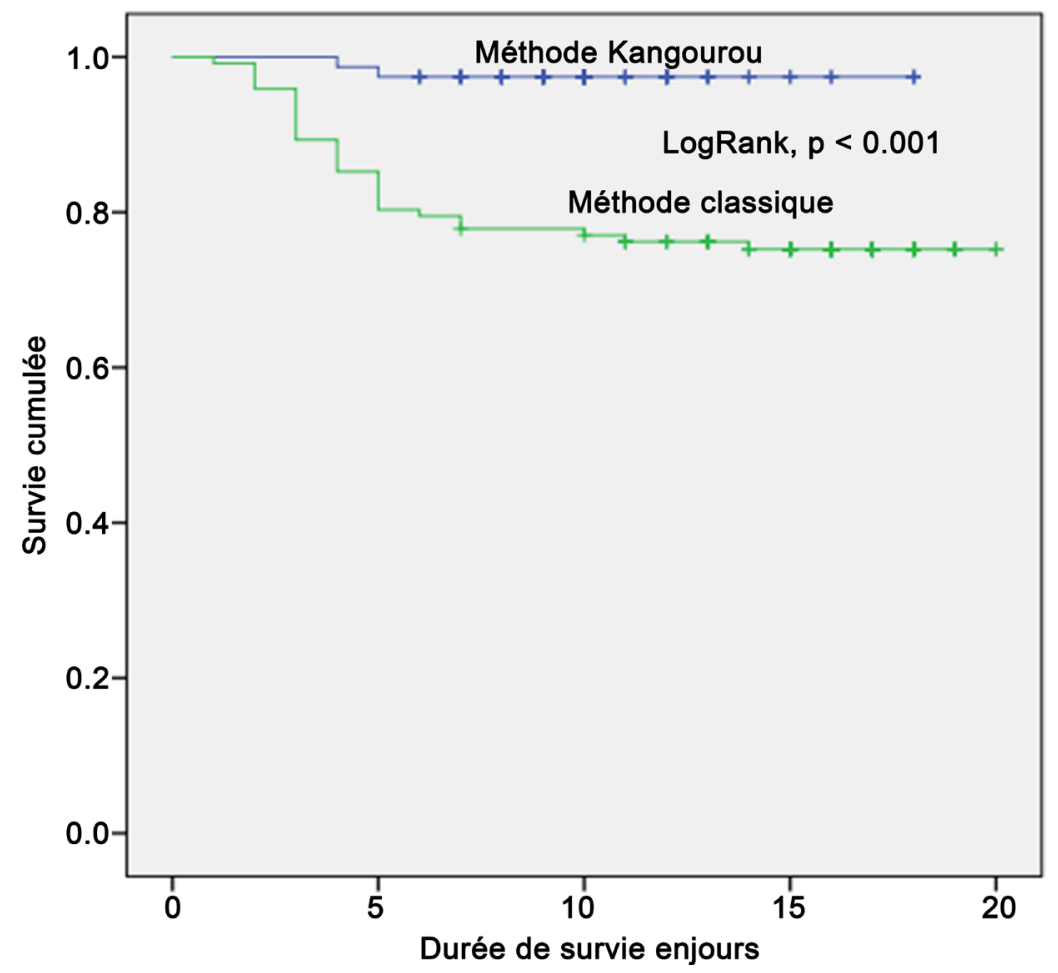

Figure 2. Proportions cumulées des prématurés des survivants (Kaplan-Meier) en fonction des méthodes d'élevage.

sous la méthode classique était, respectivement, de 16(EIQ: 15-16) et 10(EIQ: 9-10) mois. L'incidence de décès des prématurés sous méthode Kangourou était de 0.3 cas pour 100P-J par contre celle des prématurés sous méthode classique était de 1.9 cas pour $100 \mathrm{P}-\mathrm{J}(\mathrm{p}<0.001)$.

\section{4) Facteurs prédictifs de la mortalité des prématurés}

Il ressort du Table 4 à l'analyse multivariée que les facteurs ci-après sont prédictifs de la mortalité: un poids de naissance inférieur ou égal à $1600 \mathrm{~g}$, une alimentation mixte ou au lait artificielle, la méthode d'élevage classique, un rythme de croissance mauvais, et l'hypothermie.

\section{Discussion}

\subsection{Caractéristiques Générales de la Population D'étude}

Cette étude a mis en évidence les facteurs associés à la prise en charge de la prématurité, en évaluant notamment la prise en charge par 2 méthodes de régulation de la thermorégulation: la méthode classique par incubateur, et la méthode kangourou.

Il ressort tout d'abord une meilleure acceptation de la méthode SMK chez les femmes plus jeunes. Ce qui suggère probablement que les femmes âgées, généralement multipares, seraient plus réfractaires à la nouveauté en s'appuyant sur l'expérience empirique de leurs précédents accouchements. De même, les femmes célibataires étaient plus adhérentes que celles mariées.

Il est également ressorti que les complications étaient moindres lorsque la 
Table 4. Facteurs prédictifs de la mortalité des prématurés.

\begin{tabular}{|c|c|c|c|c|c|c|}
\hline \multirow{2}{*}{ Variables } & \multicolumn{3}{|c|}{ Analyse univariée } & \multicolumn{3}{|c|}{ Analyse multivariée } \\
\hline & $\beta$ & $\mathrm{p}$ & HR (IC95\%) & $\beta$ & $\mathrm{p}$ & HRa (IC95\%) \\
\hline \multicolumn{7}{|l|}{ Parité } \\
\hline Multipare & & & 1 & & & 1 \\
\hline Primipare & 0.963 & 0.012 & $2.62(1.76-8.95)$ & 0.286 & 0.535 & $1.33(0.54-3.28)$ \\
\hline \multicolumn{7}{|l|}{ EIG } \\
\hline$\geq 24$ mois & & & 1 & & & 1 \\
\hline$<24$ mois & 0.404 & 0.025 & $2.50(1.67-3.34)$ & -0.219 & 0.601 & $0.80(0.35-1.82)$ \\
\hline \multicolumn{7}{|l|}{$\mathrm{PN}$} \\
\hline$\geq 1600 \mathrm{~g}$ & & & 1 & & & 1 \\
\hline$<1600 \mathrm{~g}$ & 2.105 & $<0.001$ & $8.21(3.38-19.96)$ & 0.206 & 0.001 & $6.23(2.46-12.28)$ \\
\hline \multicolumn{7}{|l|}{ Alimentation } \\
\hline LM & & & 1 & & & 1 \\
\hline LA ou mixte & 2.773 & $<0.001$ & $16.01(3.83-67.00)$ & 2.484 & 0.023 & $11.98(1.40-13.53)$ \\
\hline \multicolumn{7}{|l|}{ Méthode élevage } \\
\hline Kangourou & & & 1 & & & 1 \\
\hline Classique & 2.292 & 0.002 & $9.90(2.36-41.49)$ & 1.983 & 0.010 & $7.27(1.62$ - 13.64à \\
\hline \multicolumn{7}{|l|}{ Croissance } \\
\hline Bonne & & & 1 & & & 1 \\
\hline Mauvaise & 4.692 & 0.002 & $10.12(5.66-21.05)$ & 13.156 & 0.003 & $6.85(2.32-12.25)$ \\
\hline \multicolumn{7}{|l|}{ Hypothermie } \\
\hline Non & & & 1 & & & 1 \\
\hline Oui & 3.430 & 0.005 & $10.86(2.89-17.45)$ & 9.644 & 0.032 & $5.43(1.25-11.24)$ \\
\hline
\end{tabular}

méthode SMK était celle utilisée. Par ailleurs, il ressort également que la méthode SMK était la plus utilisée chez les patientes ayant eu un accouchement par voie basse, ce qui est évident dans la mesure où la mise en œuvre était dans ce cas plus aisée.

\subsection{Eléments Cliniques Associés à la Prise en Charge}

Notre étude montre de manière significative une prise de poids meilleure des nouveau-nés par la méthode Kangourou. Cette observation a déjà été évoquée dans de précédentes études [3], notamment concernant les pays en voie de développement. En effet, les conditions complexes de prise en charge du prématuré ou du nouveau-né de faible poids de naissance sont rarement réunies dans un contexte de sous-équipement (accès à l'électricité, disponibilité des incubateurs, maintien continu des standards des soins intensifs, etc.). D'où un risque accru de déperdition de chaleur et de stagnation pondérale par la méthode classique. Parallèlement, la méthode SMK, simple et facile à mettre en œuvre, offre 
un bénéfice évident quant à la continuité des soins essentiels du nouveau-né, favorisant dès lors la prise rapide de poids.

Dans notre étude également, le nouveau-né présente moins de d'épisodes d'hypothermie que par la méthode classique. En effet, le bénéfice de la régulation de température est prouvé. Par exemple, une étude antérieure montait que pendant les périodes de contact peau contre peau, la température corporelle du nourrisson augmentait de $0.22^{\circ} \mathrm{C}$ [5]. De plus, au-delà d'une une meilleure régulation de la température, la méthode Kangourou entraine un meilleur sommeil [6] sans accentuer le risque d'infection [7] [8].

Dans notre étude, le bénéfice va plus loin, avec de manière significative moins d'épisodes d'hyperthermie par la méthode Kangourou. D'autant plus quand l'incubateur est réglé à température stable, le risque d'hyperthermie est plus grand dans la méthode classique ce qui est dangereux pour le nouveau-né si la surveillance fait défaut.

En outre, la durée de séjour est moindre pour les nouveau-nés sous SMK. Dans une autre étude, la méthode SMK n'accroît ni la fréquence ou la durée des épisodes d'apnée [9]. Il est également décrit que la méthode SMK a un effet positif sur le développement de la relation mère-enfant, encourageant un attachement plus fort [10] [11].

\subsection{Facteurs Associés à la Croissance et à la Survie des Prématurés}

Les facteurs ci-après ont été significativement associés à la bonne croissance des prématurés: un poids de naissance supérieur à 1600 gr, une alimentation au lait maternel et la prise en charge par la méthode SMK.

Une analyse Cochrane a notamment conclu à l'efficacité de la méthode SMK pour remplacer les soins habituels aux nourrissons de petit poids de naissance (poids de naissance inférieur à $2500 \mathrm{~g}$ ) en soins intensifs [12].

Pour ce qui est de l'alimentation, les bienfaits du lait maternel sont bien connus pour le nourrisson prématuré. Ils incluent une diminution de l'incidence d'infections et d'entérocolite nécrosante ainsi qu'une amélioration de la croissance et des issues neurodéveloppementales. La méthode SMK s'associe à un allaitement de plus longue durée, à des volumes plus élevés de lait exprimé, à un taux plus élevé d'allaitement exclusif et à un plus fort pourcentage d'allaitement lorsque le nourrisson prématuré obtient son congé de l'hôpital [13] [14].

Quant à la survie, elle était meilleure pour les prématurés sous méthode Kangourou que par la méthode classique dans notre étude. D’après 16 études (2518 nourrissons), dont 11 ont été menées dans des pays à faible revenu ou à revenu intermédiaire, l'analyse a conclu que la méthode SMK réduisait non seulement la mortalité au congé (RR $0.60,95 \%$ IC 0.39 à 0.93 ), mais également les maladies graves, les infections et la durée d'hospitalisation, tout en améliorant l'attachement entre la mère et le nourrisson, l'allaitement et la satisfaction de la mère. Nombreux sont ceux qui croient désormais que la méthode SMK est une intervention importante pour réduire la morbidité et la mortalité chez les nourrissons de petit poids de naissance dans les pays industrialisés, et largement applicables dans les 
pays en voie de développement [15] [16]. De plus, en rapport avec les facteurs prédictifs de la mortalité dans notre étude, à côté du poids de naissance inférieur ou égal à $1600 \mathrm{~g}$, de l'alimentation mixte ou au lait artificiel, du mauvais rythme de croissance; l'usage de la méthode classique elle-même s'avère être un facteur prédictif de la mortalité. En effet, les conditions de prise en charge du prématuré en utilisant la méthode classique dans un contexte de sous-équipement ne sont souvent pas réunies, comme la continuité de la fourniture en électricité, la performance des incubateurs qui sont souvent défectueux, voire même la permanence du personnel soignant qui est souvent débordé. Ce qui souligne davantage le fait que la méthode SMK devrait être généralisée dans un tel contexte.

\subsection{Limites de L'étude}

Cette étude n'a pas pu suivre à long terme les nouveau-nés des 2 cohortes, ce qui aurait pu mettre en évidence le développement psychomoteur de ces enfants. Néanmoins, les objectifs globaux de l'étude ont pu être atteints.

\section{Conclusion}

Il ressort de cette étude que la méthode SMK est simple et facilement applicable dans le contexte d'un milieu hospitalier sous-équipé. A tous égards, la survie des prématurés et autres nouveau-nés de faible poids de naissance est meilleure. Ce qui souligne l'intérêt d'une généralisation de cette pratique.

\section{Conflits D'intérêts}

Les auteurs ne déclarent aucun conflit d'intérêts.

\section{Qu'est ce qui est connu sur ce sujet}

- La thermorégulation est une pierre angulaire dans la prise en charge du prématuré

- La méthode Kangourou est fiable et assure une prise en charge efficiente de la croissance du prématuré

\section{Qu'est-ce que votre étude apporte de nouveau}

- Dans un contexte de sous équipement, la méthode classique de prise en charge sous incubateur, dont le management est complexe et souvent mal exécuté, peut devenir elle-même un facteur de risque de mortalité. D'où l'intérêt de généraliser la méthode Kangourou quand elle est applicable

\section{Contributions des Auteurs}

Tous les auteurs ont contribué et ont validé la conception de l'étude, la récolte des données et à l'élaboration de la version finale du manuscrit.

\section{References}

[1] OMS, UNICEF (2014) Chaque Nouveau-né: plan d'action pour mettre fin aux décès 
évitables, Résumé d'orientation. Organisation mondiale de la Santé, Genève.

[2] Lawn, J.E., Blencowe, H., Oza, S., et al. (2014) Every Newborn: Progress, Priorities, and Potential beyond Survival. The Lancet, 384, 189-205.

https://doi.org/10.1016/S0140-6736(14)60496-7

[3] Jefferies, A.L. (2012) La méthode kangourou pour le nourrisson prématuré et sa famille. Paediatrics \& Child Health, 17, 144-146. https://doi.org/10.1093/pch/17.3.144

[4] Kamaye, M., Garba, M., Sani, M.M., Alido, S., Oumarou, Z. and Amadou, A. (2017) Evaluation de la prise en charge du nouveau-né de faible poids de naissance par la méthode kangourou à la maternité Issaka-Gazoby de Niamey. Journal de Pédiatrie et de Puéricultre, 30, 113-117. https://doi.org/10.1016/j.jpp.2016.12.006

[5] Mori, R., Khanna, R., Pledge, D., et al. (2010) Meta-Analysis of Physiological Effects of Skin-to-Skin Contact for Newborns and Mothers. Pediatrics International, 52, 161-170. https://doi.org/10.1111/j.1442-200X.2009.02909.x

[6] Hurst, N.M., Valentine, C.J., Renfro, L., et al. (1997) Skin-to-Skin Holding in the Neonatal Intensive Care Unit Influences Maternal Milk Volume. Journal of Perinatology, 17, 213-217

[7] Anderson, C. (1992) Current Knowledge about Skin to Skin Kangourou Care for Preterm Infants. Journal of Perinatalogy, 11, 216-226

[8] Sloan, N.L., Camacho, L.W., Rojas, E.P., et al. (1994) Kangaroo Mother Method: Randomised Controlled Trial of an Alternative Method of Care for Stabilised Low-Birth-Weight Infants. Maternidad Isidro Ayora Study Team. Lancet, 344, 782-785. https://doi.org/10.1016/S0140-6736(94)92341-8

[9] Heimann, K., Vaessen, P., Peschgens, T., Stanzel, S., Wenzl, T.G. and Orlilowsky, T. (2010) Impact of Skin to Skin Care, Prone and Supine Positioning on Cardiorespiratory Parameters and Thermoregulation in Premature Infants. Neonatology, 97, 311-317. https://doi.org/10.1159/000255163

[10] Tessier, R., Cristo, M., Velez, S., et al. (1998) Kangaroo Mother Care and the Bonding Hypothesis. Pediatrics, 102, e17. https://doi.org/10.1542/peds.102.2.e17

[11] Colonna, F., Uxa, F., da Graca, A.M., et al. (1990) The "Kangaroo-Mother" Method: Evaluation of an Alternative Model for the Care of Low-Birthweight Newborns in Developing Countries. International Journal of Gynecology \& Obstetrics, 31, 335-912. https://doi.org/10.1016/0020-7292(90)90911-4

[12] Lawn, J.E., Mwansa-Kambafwile, J., Horta, B.L., Barros, F.C. and Cousens, S. (2010) 'Kangaroo Mother Care' to Prevent Neonatal Deaths Due to Preterm Birth Complications. International Journal of Epidemiology, 39, 1144-1154. https://doi.org/10.1093/ije/dyq031

[13] Renfrew, M.J., Craig, D., Dyson, L., et al. (2009) Breastfeeding Promotion for Infants in Neonatal Units: A Systematic Review and Economic Analysis. Health Technology Assessment, 13, 1-146. https://doi.org/10.3310/hta13400

[14] Hake-Brooks, S.J. and Anderson, G.C. (2008) Kangaroo Care and Breastfeeding of Mother-Preterm Infant Dyads 0-18 Months: A Randomized, Controlled Trial. Neonatal Network, 27, 151-159. https://doi.org/10.1891/0730-0832.27.3.151

[15] Charpak, N., Ruiz, J.G., Zupan, J., et al. (2005) Kangaroo Mother Care: 25 Years after. Acta Paediatrica, 94, 514-512. https://doi.org/10.1080/08035250510027381

[16] Barros, F.C., Bhutta, Z.A., Batra, M., et al. (2010) Global Report on Preterm Birth and Stillbirth (3 of 7): Evidence for Effectiveness of Interventions. BMC Pregnancy Childbirth, 10, S3. https://doi.org/10.1186/1471-2393-10-S1-S3 